\title{
Editorials for Journal of Biodiversity \& Endangered Species
}

\section{Sudhanshu Panda*}

GIS/Environmental Science, Institute for Environmental and Spatial Analysis, Gainesville State College, USA

Biodiversity management and endangered species protection and revival are spatial issues. Most of the data used in such management decision makings are spatial in nature. Land Cover/Land Use (LULC), soil, elevation/topography, hydrology, transportation, population density and adjacency, climate/weather, etc., are a few examples of such spatial data which directly or indirectly impact the management process. Luckily, in most of the spatial data are either developed or protocols has already been developed by researchers to develop them from remote or physically available sources. One of the most prominent three technologies in recent times is geospatial technology. The other two are nanotechnology and Biotechnology. While nanotechnology deals with small things and biotechnology deals with microorganisms, geotechnology deals with the opposite, everything large. It is humanly impossible to deal with or analyze features of larger areas (physically or qualitatively as it is in smaller fragments) for accurate management decision making. Geotechnology has the ability for accurate pixel based analysis of such larger areas using several parameters as mentioned above.

Geospatial technology includes four different technologies that are embedded together to map and analyze features on the surface of the earth for environmental management. They are Geographical Information Systems (GIS), Global Positioning Systems (GPS), Remote Sensing (RS), and Spatial Information Technology (SIT). Together these four components of geospatial technology can track, map, analyze, and disseminate the environmental management information. RS technology helps surveying the entire earth with unprecedented regularity thus any environmental or biodiversity change can be noticed or monitored. Present LULC conditions or changes can be monitored efficiently and easily. Shuttle Radar Topology Mission Satellites obtain global elevation data on a regular basis from which earth topographic changes can be monitored efficiently. In present decade, with the advent of LiDAR technology, earth elevation including tree heights can be monitored with centimeter accuracy.With high resolution imagery, animal tracking such as animal census can be performed. Recently, penguin census was conducted in Antarctica using aerial imagery. Global atmospheric conditions are monitored on hourly basis by weather satellites including the monitoring of water vapor amount in atmosphere on spatial basis. RS imagery provides information on draught, vegetation vigor, flood damage, forest fires, deforestation, and other natural disasters which directly or indirectly influences the biodiversity on the earth. GIS provides the tools to accurately map this information in both global and local scale including developing automated geospatial models for precise and proficient decision making. In recent times, most widely used GPS technology accurately tracks the position of environmental fall outs, biodiversity changes and others. SIT helps improve the Decision Support System (DSS) development and popularize these fascinating but sometime challenging to comprehend tools.

Automated geospatial models are being developed now-a-days on regular basis for such environmental management and hazard mitigation decision making due to the advancement in geospatial technologies. Few examples can bring readers attention to such incredible technologies that are making the biodiversity management and endangered species protection and revival an easy, cost effective, accurate, and efficient endeavor. Endangered species habitat suitability analyses can be conducted using spatial information like LULC, proximity to urban land, roads, water, etc., spatial extent of the forest cover, spatial weather variation, prey availability, and others. Potential conservation lands for endangered and threatened species can be determined by analyzing suitable spatial features and corresponding to that to the available conservation lands. Water quality of the water bodies can be easily be evaluated by watershed hydrologic geospatial models. Probable natural hazard locations can be scaled from very high to low so that biodiversity management can be conducted on scientific basis. Endangered animals can be tracked with GPS to study their adaptation behavior so that such favorable conditions can be created for the species survival and revival. Watershed management and other environmental management DSS are being developed on regular basis since the beginning of the millennium that uses the geospatial information technology for integrating researchers, middle managers, and the end-users for successful implementation of the management schemes. Plenty of such examples exist in present day literatures that show the importance of geospatial technology in such environmental management. Therefore, I emphasize here that all of the researchers involved in environmental management, especially biodiversity management should get familiarize with this new-age technology.

*Corresponding author: Sudhanshu Panda, GIS/Environmental Science Institute for Environmental and Spatial Analysis, Gainesville State College, USA, Tel: 678-717-3594; E-mail: Sudhanshu.Panda@ung.edu

Received November 11, 2013; Accepted November 16, 2013; Published November 22, 2013

Citation: Panda S (2013) Editorials for Journal of Biodiversity \& Endangered Species. J Biodivers Endanger Species 1: e108. doi:10.4172/23322543.1000e108

Copyright: (c) 2013 Panda S. This is an open-access article distributed under the terms of the Creative Commons Attribution License, which permits unrestricted use, distribution, and reproduction in any medium, provided the original author and source are credited. 